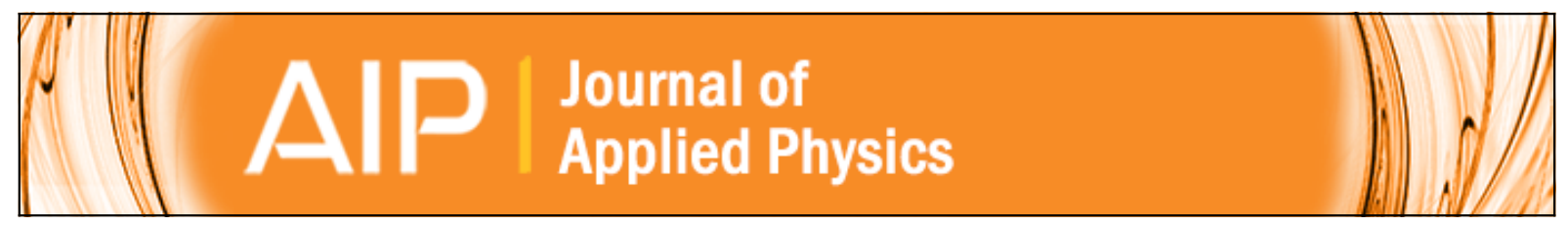

\title{
Uniaxial anisotropy and novel magnetic behaviors of CoFe 204 nanoparticles prepared in a magnetic field
}

Qian Gao, Guangyan Hong, Jiazuan Ni, Wendong Wang, Jinke Tang, and Jibao He

Citation: Journal of Applied Physics 105, 07 A516 (2009); doi: 10.1063/1.3072019

View online: http://dx.doi.org/10.1063/1.3072019

View Table of Contents: http://scitation.aip.org/content/aip/journal/jap/105/7?ver=pdfcov

Published by the AIP Publishing

\section{Articles you may be interested in}

Enhanced magnetic anisotropy in cobalt-carbide nanoparticles

Appl. Phys. Lett. 104, 023111 (2014); 10.1063/1.4862260

Role of inhomogeneous cation distribution in magnetic enhancement of nanosized $\mathrm{Ni0}$.35Zn0.65Fe2O4: A structural, magnetic, and hyperfine study

J. Appl. Phys. 114, 093901 (2013); 10.1063/1.4819809

Core/shell magnetism in $\mathrm{NiO}$ nanoparticles

J. Appl. Phys. 114, 083906 (2013); 10.1063/1.4819807

Surface contributions to the alternating current and direct current magnetic properties of oleic acid coated CoFe2O4 nanoparticles

J. Appl. Phys. 112, 123916 (2012); 10.1063/1.4770484

High temperature magnetic properties of Co1-xMgxFe2O4 nanoparticles prepared by forced hydrolysis method J. Appl. Phys. 111, 07B530 (2012); 10.1063/1.3677923

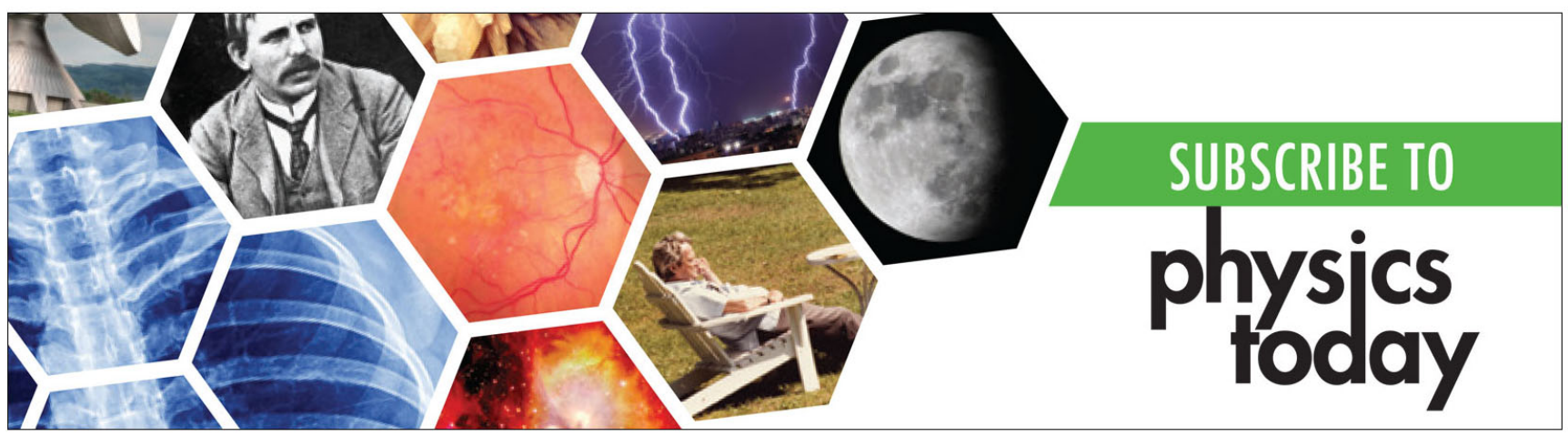




\title{
Uniaxial anisotropy and novel magnetic behaviors of $\mathrm{CoFe}_{2} \mathrm{O}_{4}$ nanoparticles prepared in a magnetic field
}

\author{
Qian Gao, ${ }^{1}$ Guangyan Hong, ${ }^{1}$ Jiazuan $\mathrm{Ni}^{1},{ }^{2}$ Wendong Wang, ${ }^{2}$ Jinke Tang, ${ }^{2, a)}$ and \\ Jibao $\mathrm{He}^{3}$ \\ ${ }^{1}$ Key Laboratory of Rare Earth Chemistry and Physics, Changchun Institute of Applied Chemistry, \\ Chinese Academy of Science, 5625 Renmin Street Changchun, Jilin 130022, China \\ ${ }^{2}$ Department of Physics and Astronomy, University of Wyoming, Laramie, Wyoming 82071, USA \\ ${ }^{3}$ Coordinated Instrumentation Facilities, Tulane University, New Orleans, Louisiana 70118, USA
}

(Presented 14 November 2008; received 22 September 2008; accepted 18 November 2008; published online 6 March 2009)

\begin{abstract}
$\mathrm{CoFe}_{2} \mathrm{O}_{4}$ nanoparticles prepared by chemical coprecipitation method in a magnetic field exhibit novel magnetic properties. The average particle diameter was about $2 \mathrm{~nm}$ and larger depending on the post annealing temperature. Magnetization measurements indicate that smaller nanoparticles are superparamagnetic above their respective blocking temperatures. In the blocked state, these nanoparticles exhibit interesting behaviors in the magnetic hysteresis measurements. Constricted, or wasp waisted with extremely narrow waist, hysteresis curves have been observed in the magnetization versus field sweeps. For larger nanoparticles, the room temperature hysteresis is typical of a ferromagnet with an open loop, but the loop closes at lower temperature. The novel magnetic behavior is attributed to the directional order of Co ions and vacancies in $\mathrm{CoFe}_{2} \mathrm{O}_{4}$ established during the coprecipitation of the nanoparticles under an applied field. (C) 2009 American Institute of Physics. [DOI: 10.1063/1.3072019]
\end{abstract}

\section{INTRODUCTION}

Induced magnetic anisotropy due to directional order has been studied since half a century ago. ${ }^{1-4}$ Directional order of like atom pairs in Co-Ni-Fe alloys, or Co in Co-ferrites, induced by magnetic annealing is responsible for the wasp waisted, or constricted, hysteresis curves. ${ }^{5,6}$ The materials show low or no hysteresis loss in low magnetic fields and exhibit large constant permeability important for applications. Most of the previous studies on $\mathrm{CoFe}_{2} \mathrm{O}_{4}$ regarding directional order have focused on bulk or single crystal materials where domain wall motion plays an important role in their magnetic properties. ${ }^{7}$ Recent advances in nanomaterial preparation have enabled researchers to prepare nanoparticles of Co-ferrites with various newly developed methods, and novel magnetic properties of $\mathrm{CoFe}_{2} \mathrm{O}_{4}$ nanoparticles have been revealed. For example, anisotropic nanoparticles were obtained after magnetic annealing, ${ }^{8}$ and shape control was achieved in the preparation of spinel Co-ferrite nanocrystals. ${ }^{9}$ Interesting magnetic hysteresis loops associated with cation distribution in spinel structure were observed in epitaxial films grown at low temperature. ${ }^{10}$ In this paper, we report an interesting hysteresis behavior of single domain $\mathrm{CoFe}_{2} \mathrm{O}_{4}$ nanoparticles prepared in an applied magnetic field.

\section{EXPERIMENTS AND RESULTS}

Cobalt ferrite nanoparticles were prepared by coprecipitation method. The details of the sample preparation is described in Ref. 11, a brief summary is given below. $\mathrm{CoCl}_{2} \cdot 6 \mathrm{H}_{2} \mathrm{O}(\mathrm{AR})$ and $\mathrm{FeCl}_{3} \cdot 6 \mathrm{H}_{2} \mathrm{O}(\mathrm{AR})$ were dissolved in

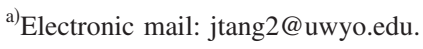

purified deoxygenated water, and a small amount of $\mathrm{HCl}(\mathrm{AR})$ was added to the solution in order to avoid hydrolyzing. The resulting solution was added dropwise into $1.5 \mathrm{M}$ $\mathrm{NaOH}(\mathrm{AR})$ solution under vigorous stirring. The last step generated an instant black precipitate. A magnetic field $H$ $=1 \mathrm{~T}$ was applied during the procedure. The supernatants were decanted and deoxygenated water added. The precipitates were obtained after washing with ethanol, centrifuging and drying at $60{ }^{\circ} \mathrm{C}$. Some of the as-prepared dried samples were annealed at 300,600 , and $800{ }^{\circ} \mathrm{C}$.

$\mathrm{X}$-ray diffraction data showed that all samples were in the spinel phase of $\mathrm{CoFe}_{2} \mathrm{O}_{4}$. The average size $d$ of the particles was estimated using Scherrer formula. $d=2,4,27$, and $40 \mathrm{~nm}$ for the as precipitated (dried at $60{ }^{\circ} \mathrm{C}$ ) sample, and samples subsequently annealed at 300,600 , and $800^{\circ} \mathrm{C}$, respectively. Transmission electron microscopy (TEM) examination confirmed such results. Figure 1 shows the TEM image of the sample annealed at $300{ }^{\circ} \mathrm{C}$, where nanoparticles of $4-5 \mathrm{~nm}$ in diameter are seen.

Figure 2 shows the field-cooled and zero-field-cooled magnetizations as a function of temperature of $\mathrm{CoFe}_{2} \mathrm{O}_{4}$ nanoparticles dried at $60{ }^{\circ} \mathrm{C}$ and subsequently annealed at $300{ }^{\circ} \mathrm{C}$. The samples are superparamagnetic above their respective blocking temperature $T_{B}=69 \mathrm{~K}$ and $149 \mathrm{~K}$ for the 60 and $300{ }^{\circ} \mathrm{C}$ samples. The inset shows the typical superparamagnetic $M$ versus $H$ curve of the nanoparticles above their blocking temperatures (measured at $300 \mathrm{~K}$ for the latter sample). Using expression $T_{B}=K V / 25 k_{B}$, and anisotropy constant $K=1.96 \times 10^{6} \times \exp \left(-1.90 \times 10^{-5} \times T_{B}^{2}\right) \mathrm{J} / \mathrm{m}^{3}$ for $\mathrm{CoFe}_{2} \mathrm{O}_{4},{ }^{12}$ the average particle diameters are found to be about 2.9 and $4.2 \mathrm{~nm}$, which are consistent with the value obtained from $\mathrm{x}$-ray diffraction and TEM. For samples an- 


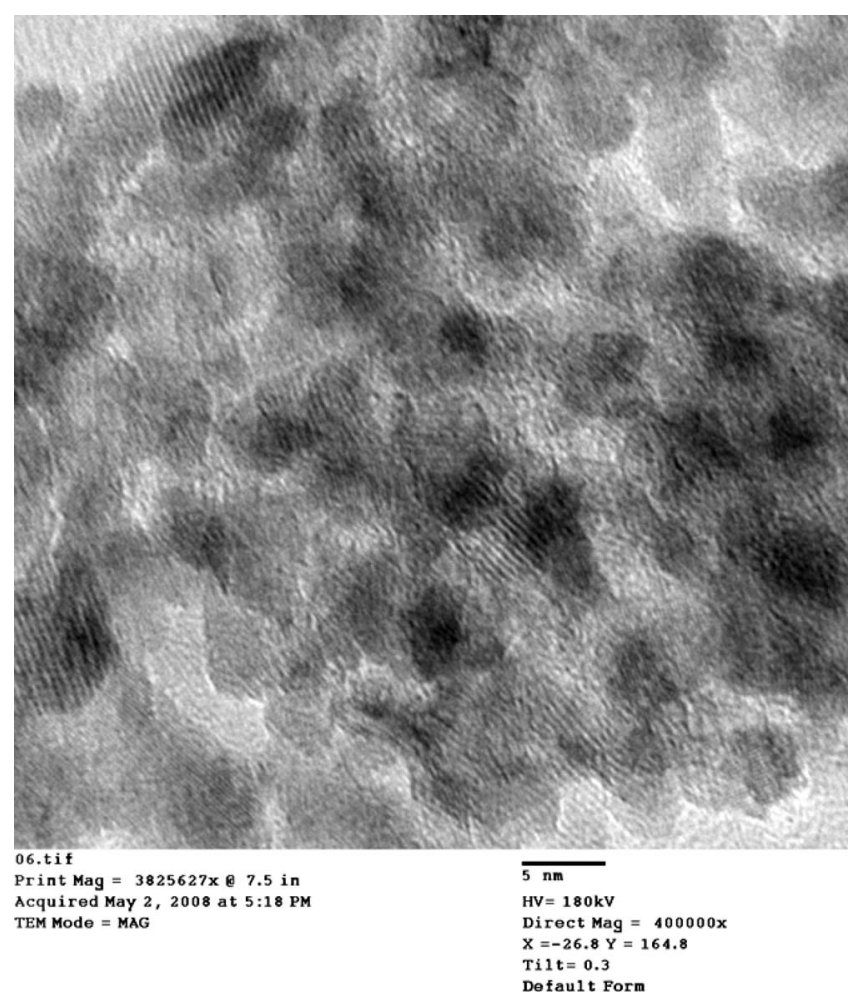

FIG. 1. TEM image of the $\mathrm{CoFe}_{2} \mathrm{O}_{4}$ nanoparticles annealed at $300{ }^{\circ} \mathrm{C}$. nealed at 600 and $800{ }^{\circ} \mathrm{C}$ (particle size of 27 and $40 \mathrm{~nm}$, respectively) ferromagnetic behavior is observed at room temperature.

In the blocked states below the blocking temperature $T_{B}$, we have observed an interesting magnetic hysteretic behavior that is not commonly seen in $\mathrm{CoFe}_{2} \mathrm{O}_{4}$ nanoparticles. An almost ideally constricted hysteresis curve is obtained at low temperature. Figure 3 shows the magnetization versus field curve of a $\mathrm{CoFe}_{2} \mathrm{O}_{4}$ sample prepared in a magnetic field and subsequently annealed at $300{ }^{\circ} \mathrm{C}$. The measurement was done at $5 \mathrm{~K}$. In the low field region, the hysteresis loop is closed and linear with a large slope. Both the coercivity and remanence are essentially zero. The ahysteretic magnetization is followed by opening of the loop in high fields. We believe this wasp-waisted hysteresis loop with extremely narrow waist should arise from the ordering of the Co ions and vacancies during the precipitation of the nanoparticles. Under the influence of the applied field, the Co ions and vacancies may order preferentially just as they do during magnetic annealing, where the annealing is carried out in an applied field. ${ }^{7}$ Studies have demonstrated that the ordering of Co ions on certain lattice sites, Co-vacancy pairs, and Co-Co pairs during magnetic annealing induces a uniaxial anisotropy along given crystallographic orientations. Such directional order is responsible for the constricted hysteresis behavior, which is explained in terms of domain wall motion and pinning by the potential wells formed by the directional

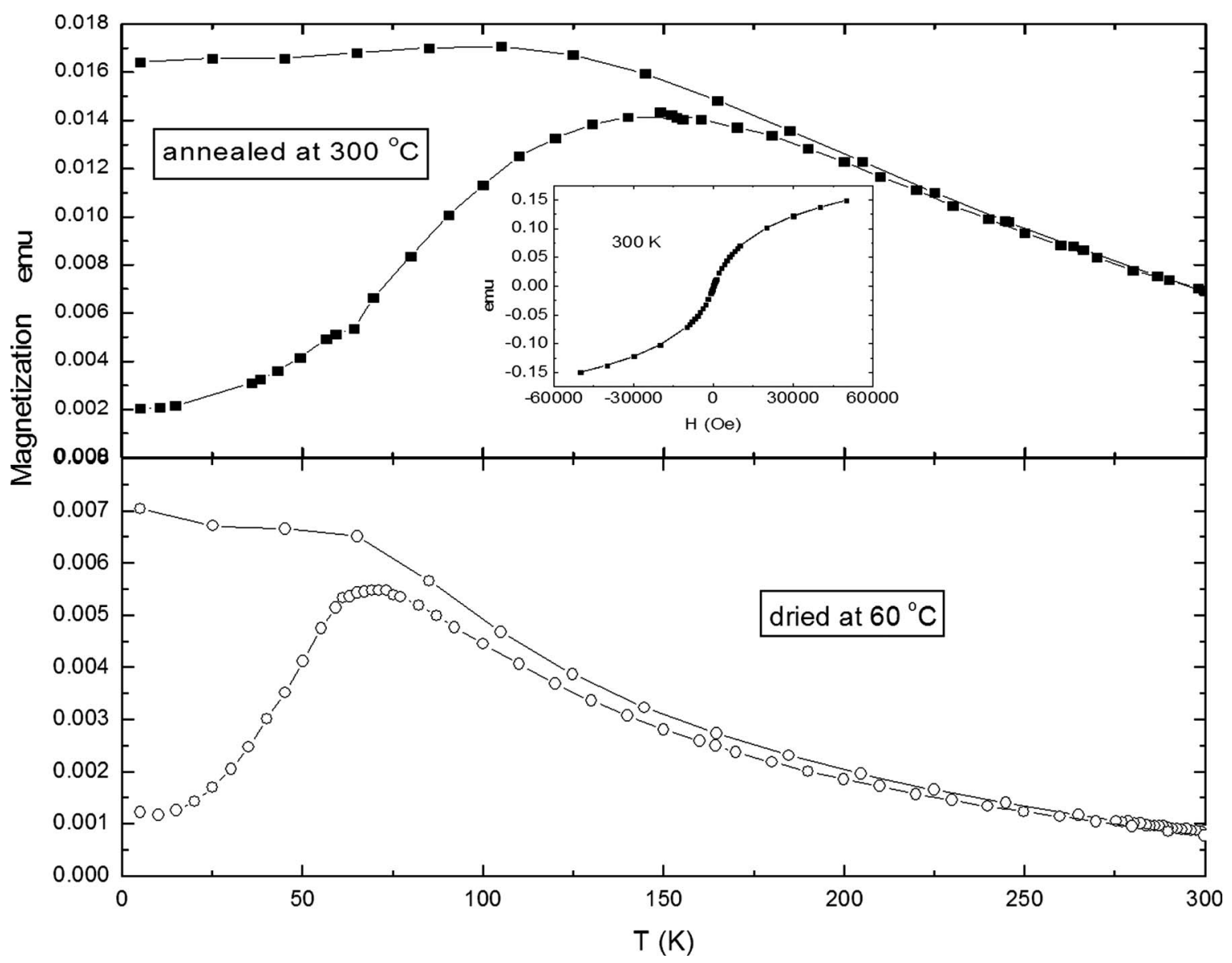

FIG. 2. Field-cooled and zero-field-cooled magnetization of $\mathrm{CoFe}_{2} \mathrm{O}_{4}$ nanoparticles dried at $60{ }^{\circ} \mathrm{C}$ (lower panel) and subsequently annealed at $300{ }^{\circ} \mathrm{C}$ (upper panel) (measuring field $H=500 \mathrm{Oe}$ ). Inset shows the typical superparamagnetic $M$ versus $H$ curve of the nanoparticles above their blocking temperatures. 


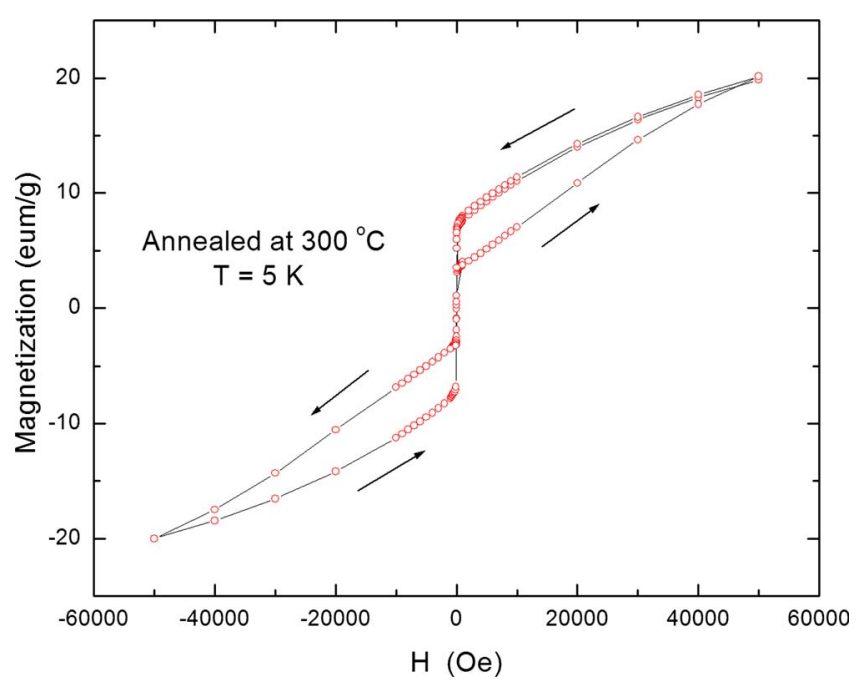

FIG. 3. (Color online) Magnetic hysteresis of a $\mathrm{CoFe}_{2} \mathrm{O}_{4}$ sample annealed at $300{ }^{\circ} \mathrm{C}$ and measured at $5 \mathrm{~K}$.

order. The difference here is that the nanoparticles under investigation are single domain. A similar uniaxial anisotropy induced during our sample preparation in magnetic field should be present. We expect an enormously large uniaxial anisotropy overcome, or play in concert with, the cubic anisotropy and be the driving force to bring the magnetic moments of the nanoparticles back to their initial directions as the field is brought back to zero. That is to bring the magnetization to the demagnetized state. Detailed study on the mechanism including the interplay between the uniaxial anisotropy and the cubic anisotropy of the $\mathrm{CoFe}_{2} \mathrm{O}_{4}$ is, however, needed to fully explain the hysteretic behavior.

For larger particles annealed at $600{ }^{\circ} \mathrm{C}$, the hysteresis at $5 \mathrm{~K}$ resembles somewhat that of a sample with ideal uniaxial anisotropy. The relative portion of the magnetization that is hysteretic is reduced as compared to the smaller particles. The magnetization shows tendency to saturate and its value is much closer to the expected $80 \mathrm{emu} / \mathrm{g}$ for $\mathrm{CoFe}_{2} \mathrm{O}_{4}$, suggesting that the larger nanoparticles are better crystallized. ${ }^{7}$ Most of the spins are aligned already near zero field, and the coercivity is essentially zero. As the temperature is increased to $300 \mathrm{~K}$, the hysteresis loop opens up with a finite coercivity (see Fig. 4). It becomes that of a typical ferromagnetic $\mathrm{CoFe}_{2} \mathrm{O}_{4}$ without induced anisotropy. The uniaxial anisotropy seems to weaken or disappear with increasing temperature rapidly, which suggests a strong temperature dependence of the anisotropy associated with the directional order mechanism and consistent with the previous reports. ${ }^{6}$ Further study is still needed to rule out the possibility that the observed hysteresis at low temperature is due to the coexistence of single domain and superparamagnetic particles in the samples, ${ }^{13}$ such a scenario is however not very likely considering the opening up of the hysteresis loop at room temperature (Fig. 4), when more superparamagnetic particles, if any, are expected than at $5 \mathrm{~K}$.

For the smallest particles (as-precipitated), saturation is far from being reached at $5 \mathrm{~T}$, typical of small magnetic particles, although the directional order induced uniaxial anisotropy exists.

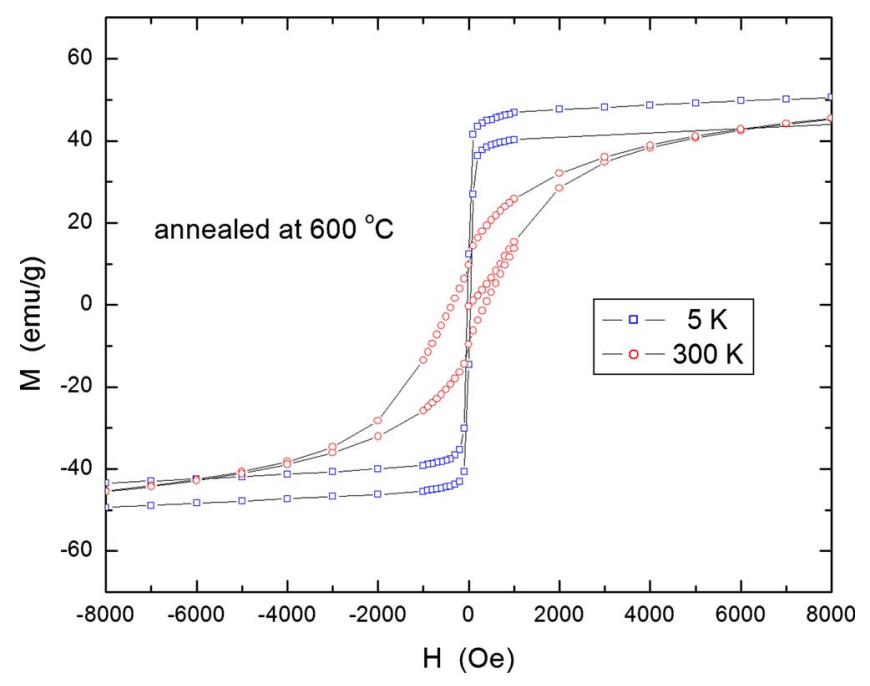

FIG. 4. (Color online) Magnetic hysteresis of $\mathrm{CoFe}_{2} \mathrm{O}_{4}$ samples annealed at $600{ }^{\circ} \mathrm{C}$, measured at 5 and $300 \mathrm{~K}$, in low field region.

\section{CONCLUSIONS}

A uniquely constricted hysteresis loop in $\mathrm{CoFe}_{2} \mathrm{O}_{4}$ nanoparticles is observed possibly due to the directional order induced during the sample preparation in an applied magnetic field. In the low field region, the magnetization has zero hysteresis loss and linear field dependence. The hysteresis loop opens up in higher field and thus exhibits a narrowwaisted wasp. This novel behavior of the magnetization in single domain nanoparticles shows similarity, but at the same time distinction, from those observed in multidomain bulk samples. Further study is needed to fully understand the phenomenon, including the various anisotropies at play as well as the order and disorder of the magnetic ions in the $\mathrm{CoFe}_{2} \mathrm{O}_{4}$ lattice of the samples.

\section{ACKNOWLEDGMENTS}

This work was supported by the NSF (Grant No. DMR0852862), the School of Energy Resources of the University of Wyoming, and Louisiana Board of Regents Support Funds.

${ }^{1}$ R. M. Bozorth, E. F. Tilden, and A. J. Williams, Phys. Rev. 99, 1788 (1955).

${ }^{2}$ S. Iida, H. Sekizawa, and Y. Aiyama, J. Phys. Soc. Jpn. 10, 907 (1941).

${ }^{3}$ R. F. Penoyer and L. R. Bickford, Jr., Phys. Rev. 108, 271 (1957).

${ }^{4}$ U. Enz, Physica 24, 68 (1958).

${ }^{5}$ J. C. Slonczewski, Phys. Rev. 110, 1341 (1958).

${ }^{6}$ S. Iida, J. Appl. Phys. 31, S251 (1960); S. Iida, H. Sekizawa, and Y. Aiyama, J. Phys. Soc. Jpn. 13, 58 (1958).

${ }^{7}$ B. D. Cullity, Introduction to Magnetic Materials, (Addison-Wesley, Reading, MA, 1972) p. 366.

${ }^{8}$ Y. Shi, J. Ding, and H. Yin, J. Alloys Compd. 308, 290 (2000).

${ }^{9}$ Q. Song and Z. J. Zhang, J. Am. Chem. Soc. 126, 6164 (2004).

${ }^{10}$ L. Horng, G. Chern, P. C. Kang, M. C. Chen, and D. S. Lee, IEEE Trans. Magn. 40, 2799 (2004).

${ }^{11}$ Q. Gao, G. Hong, J. Ni, W. Wang, J. Tang and J He (unpublished).

${ }^{12}$ H. Shenker, Phys. Rev. 107, 1246 (1957).

${ }^{13}$ L. Tauxe, T. A. T. Mullender, and T. Pick, J. Geophys. Res. 101, 571 (1996). 